\title{
Viral vector induces neurotoxicity in NHPs
}

Keiser, M.S., Ranum, P.T., Yrigollen, C.M. et al. Nat. Med. 27, 1982-1989 (2021)

RNA interference (RNAi) technologies, which work by silencing specific genes, can be delivered by adeno-associated viruses (AAV) to target mutant genes and prevent pathological gene expression. These therapies can potentially treat neuropathology, and such an approach is undergoing an early phase clinical trial for Huntington's disease. Spinocerebellar ataxia 1 (SCA1) is an adult-onset neurodegenerative disorder, caused by a CAG expansion in the Ataxin-1 gene, that results in protein aggregation, cell death and progressive problems with movement. A RNAi treatment delivering miS1, a microRNA silencing Ataxin-1, prevents and reverses behavioral deficits and neurotoxicity in mouse models of SCA1.

Having previously confirmed the feasibility and short-term safety of AAV. $\mathrm{miS1}$ in rodents and nonhuman primates (NHPs), Keiser and colleagues at The Children's Hospital of Philadelphia Research Institute investigated its long-term clinical application in NHPs. After AAV.miS1 delivery into deep cerebellar nuclei, some rhesus macaques unexpectedly developed cerebellar neurotoxicity. The researchers proceeded to dissect the root cause with next-generation sequencing, molecular and gene ontology analysis methods on the input vector, microRNA and tissue samples.

Short-read RNA sequencing revealed that cerebellar toxicity was not caused by abnormal processing of endogenous microRNAs from high artificial miS1 expression. Nanopore sequencing of the input vector showed that there was minimal but measurable cross-packaged plasmid backbone, with reads arising from the 3' inverted terminal repeat (ITR) end. New AAV constructs were trialled to elucidate if 3' ITR activity drove this toxic response. All injected vectors that allowed 3' ITR expression caused cell death in NHPs, but a new construct with minimal 3' ITR-directed expression did not. This construct, named AAV.IntmiS1, does not allow 3' ITR transcription and moves the miS1 sequence into an intron, thus retaining gene expression. None of the NHPs injected with AAV.IntmiS1 developed neurological symptoms or showed imaging pathology. While this new construct resulted in less miS1 concentrations in the brains of NHPs, efficacy remained, yet 3' ITR transcription and neuropathological readouts were importantly reduced.

These studies guided the generation of a new RNAi construct that was both effective and safe in the brains of primates. Despite long-term mouse data projecting the safety of AAV-miS1, serious side-effects transpired during advanced mammal testing. These findings confirm the need for extensive safety studies in many species when evaluating new medicines intended for human use.

\section{Ciarán Martin Fitzpatrick}

Published online: 23 December 2021 https://doi.org/10.1038/s41684-021-00905-w

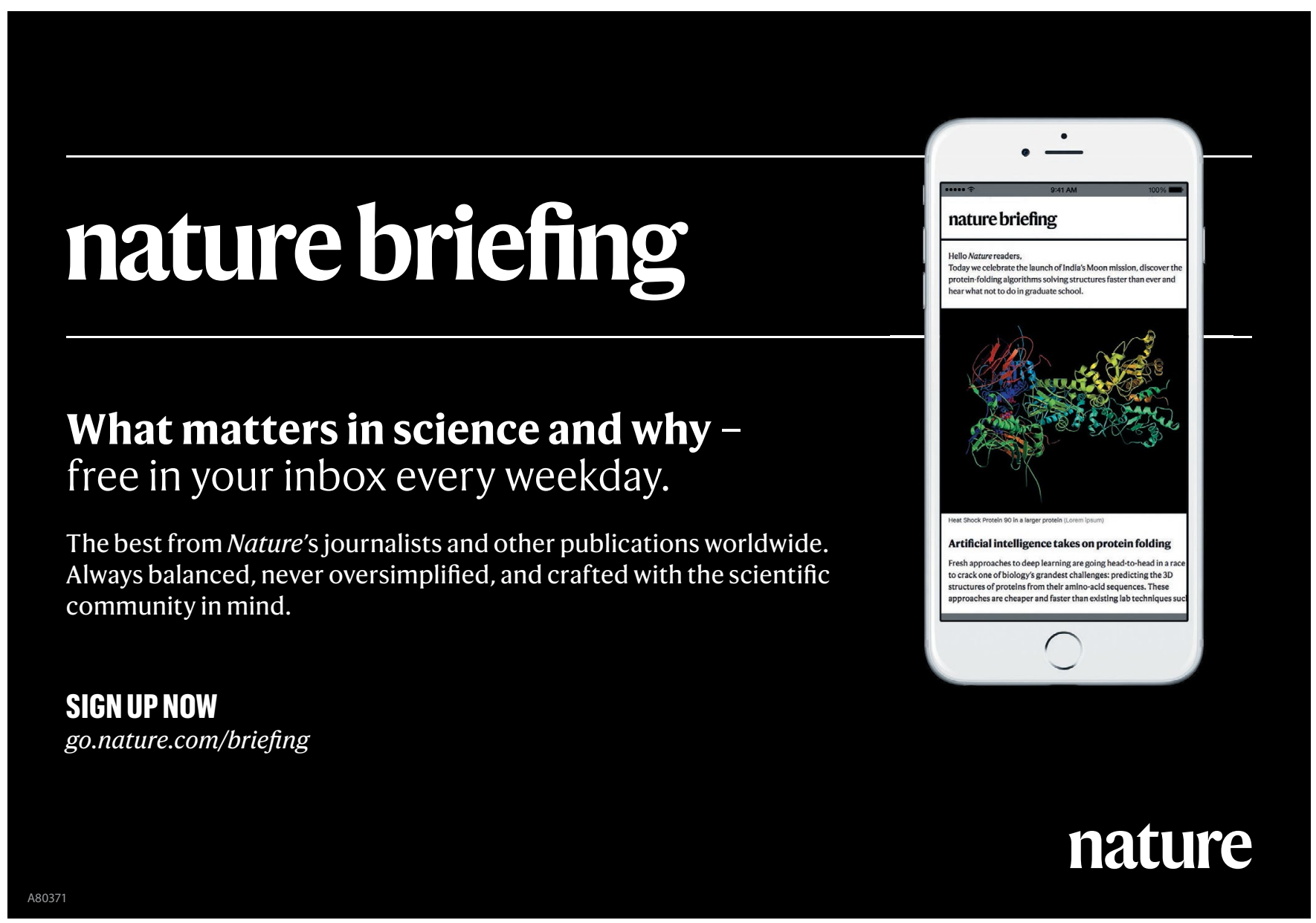

